\title{
Reply: “Adjuvant” Hyperthermic Intraperitoneal Chemotherapy: A Call to Action
}

\author{
David L. Morris, MD, PhD \\ Department of Surgery, St George Hospital and University of New South Wales, Sydney, NSW, Australia
}

I read with interest the editorial of McAuliffe and Nash. ${ }^{1}$ We believe that neither this editorial nor the paper should be seen without context. The statement that "to date no study has definitely shown that hyperthermic intraperitoneal chemotherapy (HIPEC) in combination with cytoreductive surgery (CRS) is effective for peritoneal metastases of colorectal origin..." is one that we do not agree with.

Complete CRS and HIPEC has been extensively reported to achieve a 5 -year survival hovering around $40-65 \%$ in colorectal cancer $(\mathrm{CRC})$ patients with a peritoneal carcinomatosis index $(\mathrm{PCI})<10 .^{2-4}$ The real problem is that when the PCI is beyond this, there is an associated reduction in overall survival to less than half. The majority of patients are in this category when peritoneal dissemination is diagnosed. A proposal of "adjuvant" HIPEC is, in our opinion, warranted in an attempt to improve patient outcomes and progress our knowledge and treatment of the malignancy.

Prophylactic HIPEC in high-risk CRC (pT3/pT4 and mucinous or signet ring cell histology) at the primary resection has been reported by Sammartino et al. ${ }^{5}$ In their study, peritoneal metastases and local recurrence developed significantly less often in proactively managed patients than in controls ( $4 \%$ vs. $28 \% ; p<0.03$ ), and there was significantly longer disease-free and overall survival than in the control group $(p<0.05$ and $p<0.04)$. Elias reported similar results with a planned second-look at 6 months in 41 patients with synchronous peritoneal disease, ovarian metastases, or perforation on the primary resection (high-risk). Peritoneal disease was discovered

(C) Society of Surgical Oncology 2017

First Received: 1 January 2017;

Published Online: 20 November 2017

D. L. Morris, MD, $\mathrm{PhD}$

e-mail: david.morris@unsw.edu.au intraoperatively in 56\%, without any sign of recurrence on preoperative imaging, and was treated with complete CRS/ HIPEC. The 5-year overall survival was $90 \%{ }^{6}$ A large, French, randomized, controlled trial of second-look at 6 months, which was commenced following these results, has now been completed and the outcomes will be available in early 2017. Another study in the Netherlands is currently recruiting, where HIPEC is applied synchronously or in the early postoperative setting.

We believe that the T4a group remains a real issue, which is not included in the French study; however, our Australian national funding body (NHMRC) has now twice failed to fund a trial to address these patients. We second the author's statement on the necessity of international leaders combining resources and sounding a call to action to physicians caring for CRC patients at high risk of peritoneal metastases.

\section{REFERENCES}

1. McAuliffe JC, Nash GM. "Adjuvant" hyperthermic intraperitoneal chemotherapy: a call to action. Ann Surg Oncol. 2017;24:11-2.

2. Mirnezami R, Moran BJ, Harvey K, et al. Cytoreductive surgery and intraperitoneal chemotherapy for colorectal peritoneal metastases. World J Gastroenterol. 2014;20(38):14018-32.

3. Yonemura Y, Canbay E, Ishibashi H. Prognostic factors of peritoneal metastases from colorectal cancer following cytoreductive surgery and perioperative chemotherapy. 2013. Sci World J. https://doi.org/10.1155/2013/978394.

4. Yan TD, Morris DL. Cytoreductive surgery and perioperative intraperitoneal chemotherapy for isolated colorectal peritoneal carcinomatosis: experimental or standard of care? Ann Surg. 2008;248:829-35.

5. Sammartino P, Sibio S, Biacchi D, et al. Long-term results after proactive management for locoregional control in patients with colonic cancer at high risk of peritoneal metastases. Int $J$ Colorectal Dis. 2014;29(9):1081-9.

6. Elias D, Honore C, Dumont F, et al. Results of systematic second look surgery plus HIPEC in asymptomatic patients presenting a high risk of developing colorectal peritoneal carcinomatosis. Ann Surg. 2011;254(2)289-93. 